\title{
Plantar fascia loading at different running speed: a dynamic finite element model prediction
}

\author{
Tony Lin-Wei Chen ${ }^{1}$, Duo Wai-Chi Wong ${ }^{1,2}$, Yan Wang ${ }^{1,2}$, Ming Zhang ${ }^{1,2}$ \\ ${ }^{1}$ Department of Biomedical Engineering, Faculty of Engineering, The Hong Kong Polytechnic University, Hong Kong, People's \\ Republic of China \\ ${ }^{2}$ The Hong Kong Polytechnic University Shenzhen Research Institute, Shenzhen, People's Republic of China
}

\begin{abstract}
Loads on the plantar fascia could be influenced by running speed and relate to its pathology. This study calculated and compared plantar fascia strains under different running speed conditions using a dynamic finite element foot model and computational simulations. The model was previously validated featuring twenty bones, bulk soft tissue, muscles/ligaments, and a solid part of plantar fascia. A runner performed running trials under one preferred speed (PS), two lower (PS - 10\% and PS $-20 \%$ ) and two higher (PS $+10 \%$ and PS $+20 \%$ ) speed conditions. The movement data were processed to drive musculoskeletal modelling and calculated boundary/loading conditions for the subsequent finite element analyses. The results show that peak strains of the plantar fascia increased with increasing running speed. From PS $-20 \%$ to PS $+20 \%$, peak strain in the proximal and distal fascia regions increased by $96.78 \%$ and $58.89 \%$ respectively. Running speed could directly affect plantar fascia loading, which should be considered in running regimens and rehabilitation programmes. However, prescribing speed control for runners is worth pondering as it influences the trade-off between maximum singlestep loads and loading frequency, which in coalescence determine the risk of plantar fascia injury and warranted further investigations.
\end{abstract}

KEYWORDS Plantar fascia; running; tensile strain; finite element modelling; computational simulation

CONTACT Ming Zhang ming.zhang@polyu.edu.hk

Received 26 March 2020

\section{Introduction}

Due to its known healthy benefits, running is a popular activity for many people (van Gent et al., 2007). Unfortunately, running-related injuries have remained highly prevalent as $26-75 \%$ of runners - both recreational and competitive ones - are estimated to sustain some forms of overuse injuries in a given year (Hryvniak et al., 2014). Plantar fasciitis was one of the most common foot problems occurring to runners (Taunton et al., 2002). More than two million individuals were diagnosed with plantar fasciitis in the United States annually (Roxas, 2005) and about $10 \%$ of the population were predicted to develop plantar fasciitis in their lifetime (Riddle et al., 2003).

Plantar fascia injury was primarily caused by microtears on the fascial band secondary to repeated mechanical overload and excessive strain (Cutts et al., 2012). Plantar fascia attaches to the calcaneus and the five digits on the plantar facets and regulates motions of the foot arch through passive stretching (Wearing et al., 2006). External loading on the lower limb causes structural deformation of the foot arch and strains plantar fascia. Biomechanical factors in movement strategies, e.g. running speed, have been considered one of the factors that influences the force produced through foot osteofascial structures and subsequent risk of injury (Wilk et al., 2000; Fredericson and Misra, 2007).

Running speed has been frequently modulated for load management and injuries control for runners (Hreljac et al., 2000; Hreljac, 2005). A positive relationship between ground reaction force, foot joint motion and running speed has been well established (Edwards et al., 2010; de David et al., 2015). Anecdotal information suggests that a similar relationship existed for loads on plantar fascia (Welk et al., 2015). A reduction in running speed is expected to alleviate tension on the plantar fascia and facilitate injury prevention, although evidence in the mechanical context is currently scarce. Identifying the loading patterns of the fascial band may therefore supplement the insight into the scenario.

While in-vivo measurements of the plantar fascia force were usually difficult for human locomotion, computational simulation has emerged as a substitute method for solving the problem, which allows direct estimation of internal strains on foot soft tissues during a dynamic task. The purpose of this study is to determine the influence of changing running speed on the plantar fascia loading in running. Increased running speed was hypothesised to produce increments in tensile strains on the fascia band. To answer the research question, the stance phase of running trials was simulated under five speed conditions using an established finite element foot model.

\section{Methods}

\subsection{Participant}

A healthy male (age: 30, height: $170.5 \mathrm{~cm}$, weight: 
$68.1 \mathrm{~kg}$ ) volunteered for the study. He was an experienced runner with a weekly training volume of 15 kilometres and a usual pacing of 6 minutes per kilometre. He was recruited for both computer model construction and gait analysis. The participant did not report any musculoskeletal diseases/ injuries that could influence his running performance at the time of experiment. He was fully informed of the research procedure and signed the consent form. The experimental protocol was approved by the institution (NO. HSEARS20170626003).

\subsection{Experimental procedure}

The experiment comprised several running trials under five running speed conditions: the runner's preferred speed (PS, $10 \mathrm{~km} / \mathrm{h}$ as reported by the runner), two decreased speed conditions (PS - 10\% and PS - 20\%), and two increased speed conditions (PS $+10 \%$ and $\mathrm{PS}+20 \%$ ).

The participant was given ample time to acclimatise to the running condition before the experiments. The five speed conditions were tested in a random sequence with a five-minute rest interval. Footwear was excluded from the tests so as to eliminate its effects on the running gait pattern. Data collection began with a static calibration trial that would later be used to scale an established musculoskeletal model. Afterwards, the participant ran through the motion capture region where two pairs of photoelectric cells were placed $2.6 \mathrm{~m}$ apart along the runway to monitor running speed (Hamill et al., 2014). Data generated from the running trials were considered valid when the measured running speed fell with 5\% variance of the target value and the runner's footsteps landed completely within the force platforms.

A motion capture system with eight cameras (Vicon, Oxford Metrics Ltd., Oxford, UK) and two force platforms (OR6, AMTI, Watertown, USA) were used to record marker trajectories and ground reaction force at the sampling rate of $250 \mathrm{~Hz}$ and $1000 \mathrm{~Hz}$ respectively. Thirtysix retroreflective markers were affixed to the following anatomic landmarks: acromioclavicular joints, posterior/ anterior iliac spines, greater trochanters, lateral/medial femoral epicondyles, lateral/medial malleoli, calcaneal tuberosity, the base/head of the first and fifth metatarsals, and the distal phalanx of the hallux.

\subsection{Musculoskeletal model}

A representative trial of each speed condition was selected for computational simulations. The kinematic and kinetic data were first input to the OpenSim platform (version 4.0, National Centre for Simulation in Rehabilitation Research, Stanford, USA). A generic model (John et al., 2013) with 12 rigid-body segments, 23 degrees of freedom, and 92 musculotendinous units was scaled to accommodate the mass and anthropometry of the participant. Inverse kinematics was then solved and the dynamic inconsistency was reduced by small adjustments to model mass properties (Arnold et al., 2010). The builtin tool Computed Muscle Control was implemented to compute muscle excitations that drove the desired movement patterns. Upon completing the analysis steps, forces of the external foot muscles, joint reaction force, and segmental kinematics were exported as the boundary and loading conditions for the subsequent finite element analysis.

\subsection{Finite element model}

\subsubsection{Geometry reconstruction}

A finite element model was built on the MRIs of the participant's left foot. The MRIs were acquired by a 3.0 scanner (GoldSeal Certified Signa HDxt, General Electric Company, Boston, USA) at T1 sequence, 1-mm slice interval, and $0.625 \mathrm{~mm}$ pixel size with the participant's leg fixed at neutral position by a customised ankle-foot orthosis. The image was processed to obtain the $3 \mathrm{D}$ foot geometries with an aid of segmentation software (Mimics and 3-matics, Materialise, Leuven, Belgium). The foot model was validated against a cadaveric experiment in our previous study (Chen et al., 2020). Simply speaking, the foot model was proximally fixed and sustained a pulling force of $98.1 \mathrm{~N}$ on the plantar forefoot to mimic a loading condition of plantar fascia-stretching exercise. Strains on selected sites of the fascia band were compared between our simulations and the cadaveric study. A good agreement was established between the predicted and measured strain values through a regression analysis (Chen et al., 2020).

The model included 20 bony segments, 26 ligaments, 9 intrinsic foot muscles, and 11 extrinsic foot muscles, as shown in Figure 1 (Chen et al., 2020). The bulk soft tissue was modelled by a cluster of smooth particle hydrodynamics (SPH) particles encapsulated in a shell unit using the cohesive property. The SPH particles were shown to validly incorporate themselves into regular deformable elements in the finite element domain (Chen, Wong, et al., 2019) and had the merits of solving high-impact problems (Zhang and Batra, 2009). The shell unit consisted of interior and exterior surfaces representing the periosteum layer (tied to the bony structures) and skin layer (in contact to the ground) respectively. Ligaments and intrinsic foot muscles were modelled as truss units while the extrinsic foot muscles were embodied by mechanical connectors. Both bones and plantar fascia were reconstructed as 3D solids. The geometry and placement of the plantar fascia were determined by its manifests in MRIs and the human anatomy atlas (Standring, 2020) using computer-aided design software (Solidworks v2014, Dassault Systèmes, MA, USA). From the origin to distal end, the thickness of the fascial bands gradually reduced from $3.0 \mathrm{~mm}$ to 1.3 $\mathrm{mm}$. The plantar fascia was tied to the inferior calcaneus proximally and to the heads of five phalanges distally. 


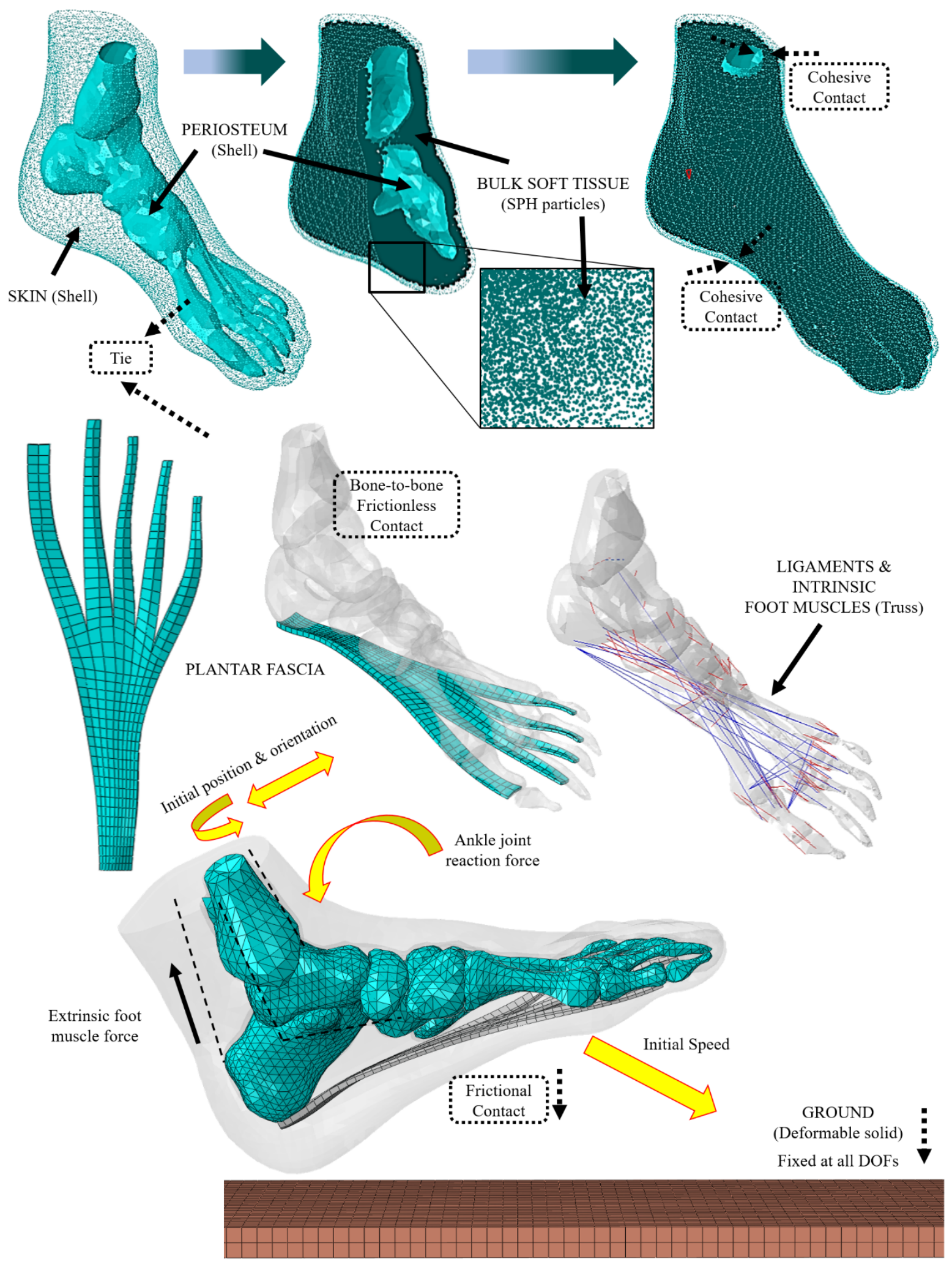

Figure 1. Outline of the finite element modelling and the boundary/loading conditions. Solid black arrows denote the names of the parts. Solid maize arrows represent boundary/loading conditions. Dashed frames denote the interactions among parts. The bulk soft tissue was modelled as SPH particle elements and encapsulated in a shell unit that possessed an interior profundal fascia layer (tied to the skeletal structures) and exterior skin layer (in contact with the fixed ground). The foot segments were connected by ligaments (truss unit), intrinsic foot muscles (truss unit), and the plantar fascia (three-dimensional solid). Three-dimensional ankle joint reaction force, extrinsic foot muscle force, and initial transitional velocity were applied to the model to drive the simulation. DOFs = degrees of freedom; $\mathrm{SPH}=$ smoothed-particle hydrodynamics. 


\subsubsection{Material properties and mesh creation}

All model components were assumed linearly elastic and isotropic except that plantar fascia was assumed to be hyperelastic and isotropic. Parameters for the material equations were acquired from the literature (Table 1). The model mesh was created by Abaqus v6.14 (Simulia, Dassault Systèmes, RI, USA). Previous mesh convergence tests had determined an optimal global mesh size of $3.5 \mathrm{~mm}$ for the osseous and soft tissue components (Chen, Wong, et al., 2019). A frictionless contact algorithm was used for bone-to-bone interface to mimic the function of cartilages (Athanasiou et al., 1998). The interaction between the skin and the ground plate was "hard" contact with a friction coefficient of 0.6 (Zhang and Mak, 1999).

\subsubsection{Boundary and loading conditions}

Boundary and loading conditions for the finite element analyses were input as a tabulated time-series matrix from preceding musculoskeletal modelling. Concentric connector force was applied to the slip-ring connectors to simulate extrinsic foot muscle force. Three-dimensional ankle joint reaction force was loaded on the ankle surface of the talus (Figure 1). The whole foot model was initially positioned and oriented corresponding to the instant before foot strike. A pre-defined velocity was also assigned to the whole foot model. Gravity was enabled throughout the simulation steps using the force/mass ratio of 9.8 .

\subsection{Simulation solver and data output}

The Abaqus dynamic explicit solver (version 6.14, Simulia, Dassault Systèmes, RI, USA) was used to perform running simulations for each speed condition. To avoid artefacts of extreme deformation, principal tensile strain was reported for the central fascial band excluding regions that were attached to the bones at the proximal and distal ends. The central plantar fascia was equally divided into three portions from proximal to distal. Principal tensile strain was averaged across all elements of each portion and the mean values were compared across the conditions. Strain contours of the plantar fascia were also plotted.

\section{Results}

Figure 2 displays the principal tensile strains on the plantar fascia (from the proximal to the distal regions) as a function of percentile stance phase. The fascial band was increasingly loaded after initial contact and maximally loaded during the mid-to-terminal stance phase (50-90\% stance). The results show that running speed posed an effect on fascia strain level. Compared with the PS condition, peak strain in the proximal fascia region decreased by $7.89 \%$ and $4.32 \%$ in the PS - $20 \%$ and PS - $10 \%$ conditions, and increased by $13.74 \%$ and $17.08 \%$ in the PS $+10 \%$ and PS $+20 \%$ conditions. Likewise, peak strain in the distal fascia region decreased by $3.95 \%$ and $5.52 \%$ in the PS $-20 \%$ and PS - $10 \%$ conditions, and increased by $15.65 \%$ and $23.17 \%$ in the PS $+10 \%$ and PS $+20 \%$ conditions. Peak strains in the mid fascia region were most reduced in the PS - $20 \%$ condition (by $20.45 \%$ ) but roughly comparable between the PS and two acceleration conditions. Figure 3 shows the strain maps of the plantar fascia when it was most loaded at late stance for the five speed conditions. As running speed increased, there was clearly a wider distribution of warncolour coded regions in the fascia band, which indicated a higher strain level according to the colour atlas.

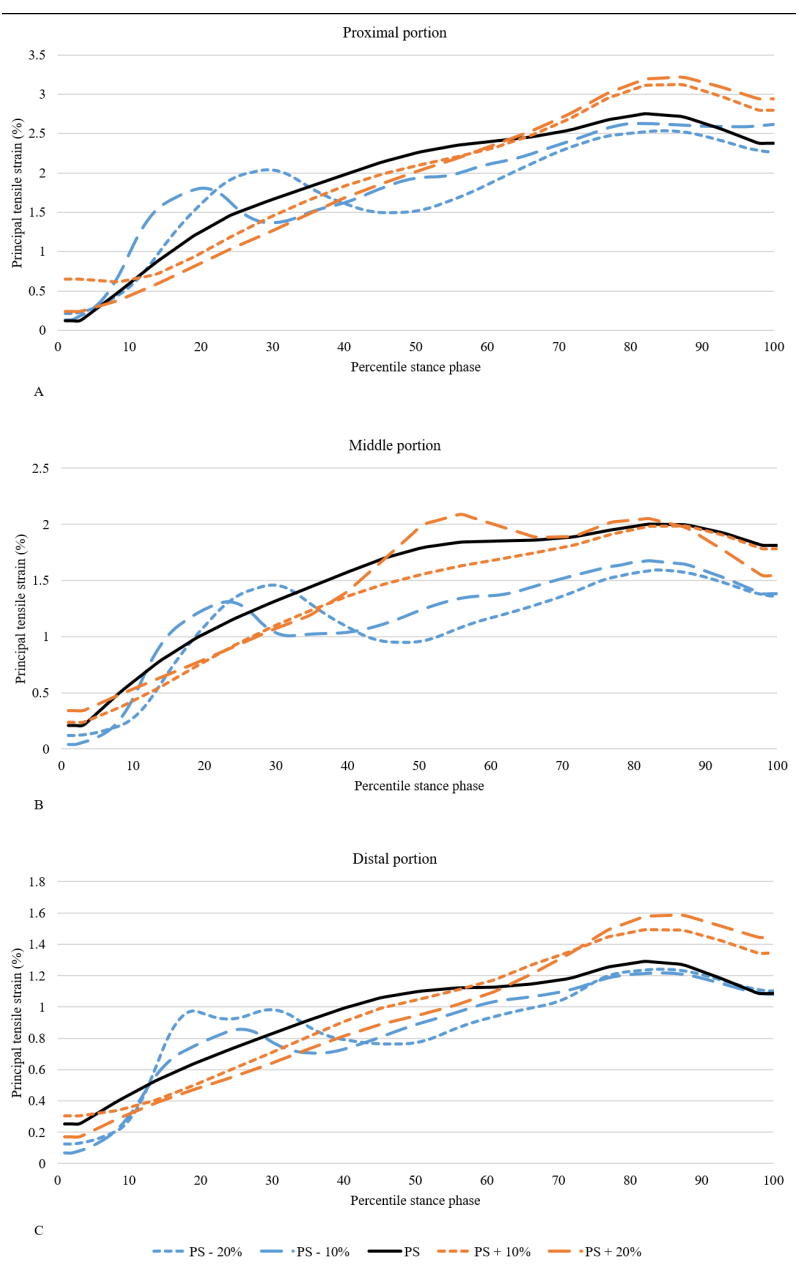

Figure 2. Changes in tensile strains on the three portions of the plantar fascia as a function of percentile running stance. A: the proximal portion; B: the middle portion; $\mathrm{C}$ : the distal portion. 


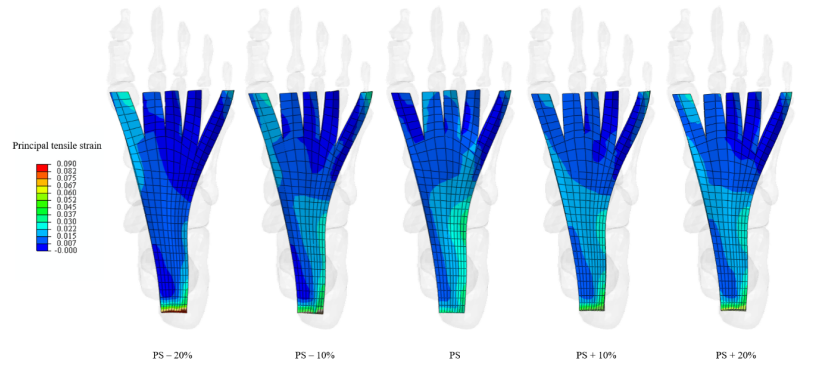

Figure 3. Strain maps of the plantar fascia for the five running speed conditions. The contour plots were extracted at the instant of maximal strain for each condition. The images are colour-coded based on the distribution of principal tensile strains. The highest strain is marked in red, and the lowest strain is marked in blue. Regions of the plantar fascia tied to the bony segments are removed from display.

\section{Discussion}

Running towards higher speeds increases forces that apply to the lower limb (Schache et al., 2011; de David et al., 2015). Increased mechanical burden to the foot was believed to tighten the plantar foot soft tissues and exacerbate foot problems (Chen, Agresta, et al., 2019). However, there is limited quantitative evidence reflecting tension in the plantar fascia in running tasks, which could be associated with injury risk, fitness, and rehabilitation program. For this reason, we calculated the plantar fascia strains during the stance of running gait and examined how it could be influenced by different running speeds. In accordance with our hypothesis, tensile strains on the fascia band (from proximal to distal portions) increased as the runner accelerated during the running trials. The increments in fascial strains were most distinct in the transition from mid- to terminal-stance.

Similar to our previous work, the magnitude of peak fascial strain (1.22-3.22\%) calculated in the current study agreed with a normal strain range (1.51-2.73\%) for common activities, such as walking (Erdemir et al., 2004; McDonald et al., 2016), but apparently fell short to those (14.52\%) reported by Chen (Chen et al., 2014). In Chen's study, the effects of foot muscles and other planter soft tissues were omitted, which resulted in a large portion $(20.1 \%)$ of the total foot loading solely sustained by the plantar fascia. This percentage of load sharing was considered to be overestimated because the yield strain and force for the dissected plantar fascia specimen were around 15\% (Butler et al., 1986) and 1189 N (Kitaoka et al., 1994) respectively. Reaching over $90 \%$ of strength limit of the fascia material during a single walking step was physically improbable. The discrepancies in reported strain level of the plantar fascia among the studies reflect the existence of the synergic effects of other plantar foot soft tissues for foot supports (Kirby, 2017). Our predicted timing of peak fascial strain (80-90\% stance phase) was in line with those (80-83.3\% stance phase) of both Chen's (Chen et al., 2014) and Erdemir's (Erdemir et al., 2004). During the period, the plantar fascia was considered most tensioned by the activated windlass and turned the foot arch into a rigid lever for propelling the body forward (Bolgla and Malone, 2004).

In line with previous studies (Hamill et al., 1983; Edwards et al., 2010; Schache et al., 2011; de David et al., 2015; Boey et al., 2017), the increased fascia strain from slow to fast running conditions was probably attributed to change in force transmission along the lower leg. Without adjusting the landing strategy such as gait retraining, runners usually experienced increased impacts on the lower leg to cope with shock absorption in fast running. Increased running speed was associated with larger ground reaction force (Hamill et al., 1983; Nilsson and Thorstensson, 1989) and tibial deceleration (Boey et al., 2017). During the stance phase, the two forces apply to the base and the apex of the foot arch and form a three-point bending motion to the foot (Lieberman, 2012; Perl et al., 2012). Increments in both forces further deform the foot arch construct and bring in tension to the plantar connective tissues (Chen, Agresta, et al., 2019). In this study, the fascia strain increased by around $4 \%$ when running speed increased by $20 \%$. These changes, though seemingly small in magnitude, were reportedly equivalent to windlassing the fascia band by dorsiflexing the first metatarsophalangeal joint by around $15^{\circ}$ (Carlson et al., 2000). A slight reduction in fascia strains appeared to improve foot joint motions and benefit runners with constrained foot mobility because of a tightened fascia band (Irving et al., 2006).

To avoid possible injury risk, research suggest decreasing gait speed as a means of controlling loading to the foot soft tissues and benefiting symptomatic runners (Robbins and Maly, 2009). However, pathomechanics of plantar fasciitis resembles the procedure for material fatigue (Chen, Wong, et al., 2019). It means that both loading magnitude and accumulated loading cycles determine the onset of injuries. Concerns were raised: reducing running speed but maintaining running volume could expose runners to prolonged loads and increased loading frequency on vulnerable anatomic sites (Edwards et al., 2010). Though it is still unclear how changes in running speed and cadence would integrate to affect the injury profile over accumulated mileages, one should be careful when prescribing slow running as a management strategy, especially when repeated loading is considered (de David et al., 2015).

Among the limitations of our study are the simplification made in the modelling process. For example, the material properties were idealised as linearly elastic despite the fact that they could possess hyperelastic or viscoelastic behaviour. The assumptions may misestimate joint stiffness of the model and loading response of the soft tissues, while it remains common practice in finite element 
analysis to compromise computational power. Additionally, the single-subject design was a major consideration when interpreting the results. A representative model participant with reasonable sets of loading conditions was selected to address the concern about external validity (Wong et al., 2018). However, this approach is still weak in generalising the outcomes to the target population, especially in the context of movement science where both anthropometry and gait pattern vary among individuals.

\section{Conclusion}

Loadings on the plantar fascia during the running stance phase was influenced by running speed. Running faster increased the peak tensile strains of the plantar fascia for both the proximal and distal fascial regions. These changes were the most prominent during the transition from heel-off to push-off. The results suggest that running speed could directly affect the tension and thus the force applied onto the plantar fascia, which should be considered in running regimen and rehabilitation programmes. Controlling running speed for runners, however, should be carefully reviewed before prescription. Further studies should be conducted to examine the integration or the tradeoff among the maximum loads, cadence and accumulated mileage on the progression of plantar fascia injury.

\section{Acknowledgements}

This work was supported by the Hong Kong Research Grant Council under Grant number PolyU152065/17E, and the National Natural Science Foundation of China under Grant numbers 11732015 and 11972315.

\section{Notes on contributors}

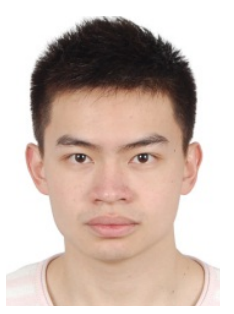

Dr Tony Lin-Wei Chen received his Ph.D. in Biomedical Engineering in 2020 from The Hong Kong Polytechnic University. He received his B.Sc. degree in Medicine and M.Phil. degrees in Orthopedic and Traumatology in 2011 and 2014 respectively from Guangzhou University of Traditional Chinese Medicine. He is currently a postdoctoral fellow working on computational simulation of running sports. His current research interests include the use of multiple modelling techniques to study sport biomechanics and injured gait patterns.

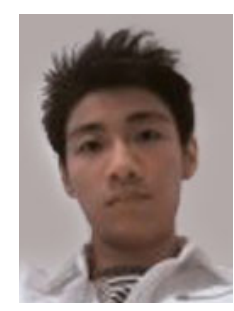

Dr Duo Wai-Chi Wong received his B.Sc., M.Phil. and Ph.D. degrees in Biomedical Engineering in 2007, 2010 and 2014 respectively from The Hong Kong Polytechnic University. He is currently a research fellow and was a postdoctoral fellow at the Department of Biomedical Engineering of The Hong Kong Polytechnic University in 2016. He is dedicated to the biomechanical simulation of orthopaedic surgery. His current research interests include computational biomechanics and sport biomechanics, particularly finite element analysis.

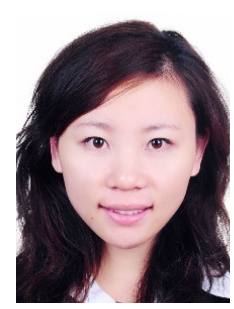

Dr Yan Wang received her Ph.D. in Biomedical Engineering in 2016 from The Hong Kong Polytechnic University. She received her M.Phil. and B.Eng. in Mechanical Engineering from Shandong University in 2011 and 2008 respectively. She is currently a research fellow at the Department of Biomedical Engineering, The Hong Kong Polytechnic University. Her research focuses on computational simulation of ankle arthrodesis and arthroplasty. She is also working on human movement analysis on flatfoot patients and foot orthotic design.

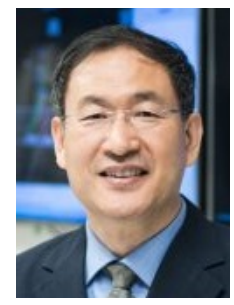

Dr Ming Zhang received his B.Sc. in automation control engineering and M.Sc. in mechanical engineering, from the Beijing Institute of Technology in 1982 and 1987 respectively. He graduated with a Ph.D. in medical engineering from King's College, University of London in 1995. He is currently Professor of Biomedical Engineering and Director of the Research Center for Musculoskeletal Bioengineering. Prof Zhang is a Councillor of the World Council of Biomechanics (WCB), President-Elect of the World Association for Chinese Biomedical Engineers (WACBE), Chair of the Chinese Society of Rehabilitation Engineering under both the Chinese Society of Biomedical Engineering and Chinese Rehabilitation Devices Association, a Committee member of the Chinese Society of Biomechanics, a Committee member of the Biomedical Division of The Hong Kong Institution of Engineers, and a Standing Council Member of the Chinese Society of Biomedical Engineering and the China Rehabilitation Devices Association. He serves as a Proposal Selection Panel Member for NSFC China and the Hong Kong Research Grant Council. He also serves as an editorial board member for a few academic journals and chaired a number of conferences. 


\section{References}

[1] Arnold EM, Ward SR, Lieber RL and Delp SL (2010). A model of the lower limb for analysis of human movement. Annals of Biomedical Engineering, 38(2), pp. 269-279.

[2] Athanasiou KA, Liu GT, Lavery LA, Lanctot DR and Schenck RC (1998). Biomechanical topography of human articular cartilage in the first metatarsophalangeal joint. Clinical Orthopaedics and Related Research, (348), pp. 269-281.

[3] Bayraktar HH, Morgan EF, Niebur GL, Morris GE, Wong EK and Keaveny TM (2004). Comparison of the elastic and yield properties of human femoral trabecular and cortical bone tissue, Journal of Biomechanics. 37(1), pp. 27-35.

[4] Boey H, Aeles J, Schütte K and Vanwanseele B (2017). The effect of three surface conditions, speed and running experience on vertical acceleration of the tibia during running. Sports Biomechanics, 16(2), pp. 166-176.

[5] Bolgla LA and Malone TR (2004). Plantar fasciitis and the windlass mechanism: a biomechanical link to clinical practice. Journal of Athletic Training, 39(1), pp. 77-82.

[6] Butler DL, Kay MD and Stouffer DC (1986). Comparison of material properties in fascicle-bone units from human patellar tendon and knee ligaments. Journal of Biomechanics, 19(6), pp. 425-432.

[7] Carlson RE, Fleming LL and Hutton WC (2000). The biomechanical relationship between the tendoachilles, plantar fascia and metatarsophalangeal joint dorsiflexion angle. Foot \& Ankle International, 21(1), pp. 18-25.

[8] Chen TL-W, Agresta CE, Lipps DB, Provenzano SG, Hafer JF, Wong DW-C, Zhang M and Zernicke RF (2019). Ultrasound elastographic assessment of plantar fascia in runners using rearfoot strike and forefoot strike. Journal of Biomechanics, 89, pp. 6571.

[9] Chen TL-W, Wong DW-C, Peng Y and Zhang M (2020). Prediction on the plantar fascia strain offload upon Fascia taping and Low-Dye taping during running. Journal of Orthopaedic Translation, 20, pp. 113-121.

[10] Chen TL-W, Wong DW-C, Wang Y, Lin J and Zhang M (2019). Foot arch deformation and plantar fascia loading during running with rearfoot strike and forefoot strike: A dynamic finite element analysis. Journal of Biomechanics, 83, pp. 260-272.

[11] Chen Y-N, Chang C-W, Li C-T, Chang C-H and Lin C-F (2014). Finite element analysis of plantar fascia during walking: a quasi-static simulation. Foot \& ankle international, 36(1), pp. 90-97.

[12] Cheung JT-M and Zhang M (2005). A 3-dimensional finite element model of the human foot and ankle for insole design. Archives of Physical Medicine and Rehabilitation, 86(2), pp. 353-358.
[13] Cook CS and McDonagh MJN (1996). Measurement of muscle and tendon stiffness in man, European Journal of Applied Physiology and Occupational Physiology. 72(4), pp. 380-382.

[14] Cutts S, Obi N, Pasapula C and Chan W (2012). Plantar fasciitis. The Annals of The Royal College of Surgeons of England, 94(8), pp. 539-542.

[15] de David AC, Carpes FP and Stefanyshyn D (2015). Effects of changing speed on knee and ankle joint load during walking and running. Journal of Sports Sciences, 33(4), pp. 391-397.

[16] Davis WH, Sobel M, DiCarlo EF, Torzilli PA, Deng X, Geppert MJ, Patel MB and Deland J (1996). Gross, histological, and microvascular anatomy and biomechanical testing of the spring ligament complex. Foot \& ankle international, 17(2), pp. 95-102.

[17] Edwards WB, Taylor D, Rudolphi TJ, Gillette JC and Derrick TR (2010). Effects of running speed on a probabilistic stress fracture model. Clinical Biomechanics, 25(4), pp. 372-377.

[18] Erdemir A, Hamel AJ, Fauth AR, Piazza SJ and Sharkey NA (2004). Dynamic loading of the plantar aponeurosis in walking. J Bone Joint Surg Am, 86(3), pp. 546-552.

[19] Fredericson M and Misra AK (2007). Epidemiology and aetiology of marathon running injuries. Sports Medicine, 37(4-5), pp. 437-439.

[20] van Gent RN, Siem D, van Middelkoop M, van Os AG, Bierma-Zeinstra SMA and Koes BW (2007). Incidence and determinants of lower extremity running injuries in long distance runners: a systematic review. British Journal of Sports Medicine, 41(8), pp. 469-480; discussion 480.

[21] Hamill J, Bates BT, Knutzen KM and Sawhill JA (1983). Variations in ground reaction force parameters at different running speeds. Human Movement Science, 2(1), pp. 47-56.

[22] Hamill J, Gruber AH and Derrick TR (2014). Lower extremity joint stiffness characteristics during running with different footfall patterns. European Journal of Sport Science, 14(2), pp. 130-136.

[23] Hreljac A (2005). Etiology, prevention, and early intervention of overuse injuries in runners: a biomechanical perspective. Physical Medicine and Rehabilitation Clinics of North America, 16(3), pp. 651-667, vi.

[24] Hreljac A, Marshall RN and Hume PA (2000). Evaluation of lower extremity overuse injury potential in runners. Medicine and Science in Sports and Exercise, 32(9), pp. 1635-1641.

[25] Hryvniak D, Dicharry J and Wilder R (2014). Barefoot running survey: Evidence from the field. Journal of Sport and Health Science, 3(2), pp. 131-136.

[26] Hurschler C, Vanderby R, Martinez DA, Vailas AC and Turnipseed WD (1994). Mechanical and biochemical analyses of tibial compartment fascia in chronic compartment syndrome. Annals of Biomedical Engineering, 22(3), pp. 272-279. 
[27] Irving DB, Cook JL and Menz HB (2006). Factors associated with chronic plantar heel pain: a systematic review. Journal of Science and Medicine in Sport, 9(1-2), pp. 11-22.

[28] John CT, Anderson FC, Higginson JS and Delp SL (2013). Stabilisation of walking by intrinsic muscle properties revealed in a three-dimensional muscle-driven simulation. Computer Methods in Biomechanics and Biomedical Engineering, 16(4), pp. 451-462.

[29] Kirby KA (2017). Longitudinal arch load-sharing system of the foot. Revista Española de Podología, 28(1), pp. e18-e26.

[30] Kitaoka H B, Luo Z P, Growney E S, Berglund L J and An K N (1994). Material properties of the plantar aponeurosis, Foot \& Ankle International, 15(10), pp. 557-560.

[31] Kura H, Luo Z-P, Kitaoka HB, Smutz WP and An K-N (2001). Mechanical behavior of the Lisfranc and dorsal cuneometatarsal ligaments: in vitro biomechanical study. Journal of orthopaedic trauma, 15(2), pp. 107-110.

[32] Ledoux WR and Blevins JJ (2007). The compressive material properties of the plantar soft tissue. Journal of Biomechanics, 40(13), pp. 2975-2981.

[33] Lieberman DE (2012). What we can learn about running from barefoot running: an evolutionary medical perspective. Exercise and Sport Sciences Reviews, 40(2), pp. 63-72.

[34] McDonald KA, Stearne SM, Alderson JA, North I, Pires NJ and Rubenson J (2016). The role of arch compression and metatarsophalangeal joint dynamics in modulating plantar fascia strain in running. PloS One, 11(4), p. e0152602.

[35] Milz P, Milz S, Steinborn M, Mittlmeier T, Putz R and Reiser M (1998). Lateral ankle ligaments and tibiofibular syndesmosis. 13-MHz high-frequency sonography and MRI compared in 20 patients. Acta Orthopaedica Scandinavica, 69(1), pp. 51-55.

[36] Nilsson J and Thorstensson A (1989). Ground reaction forces at different speeds of human walking and running. Acta Physiologica Scandinavica, 136(2), pp. 217-227.

[37] Pailler-Mattei C, Bec S and Zahouani H (2008). In vivo measurements of the elastic mechanical properties of human skin by indentation tests. Medical Engineering \& Physics, 30(5), pp. 599-606.

[38] Perl DP, Daoud AI and Lieberman DE (2012). Effects of footwear and strike type on running economy. Medicine \& Science in Sports \& Exercise, 44(7), pp. 1335-1343.

[39] Riddle DL, Pulisic M, Pidcoe P and Johnson RE (2003). Risk factors for plantar fasciitis: a matched case-control study. The Journal of Bone and Joint Surgery. American Volume, 85-A(5), pp. 872-877.
[40] Robbins SMK and Maly MR (2009). The effect of gait speed on the knee adduction moment depends on waveform summary measures. Gait \& Posture, 30(4), pp. 543-546.

[41] Roxas M (2005). Plantar fasciitis: diagnosis and therapeutic considerations. Alternative Medicine Review: A Journal of Clinical Therapeutic, 10(2), pp. 83-93.

[42] Schache AG, Blanch PD, Dorn TW, Brown NAT, Rosemond D and Pandy MG (2011). Effect of running speed on lower limb joint kinetics. Medicine and Science in Sports and Exercise, 43(7), pp. 12601271.

[43] Siegler S, Block J and Schneck CD (1988). The mechanical characteristics of the collateral ligaments of the human ankle joint. Foot \& ankle, 8(5), pp. 234242.

[44] Standring S (2020). Gray's anatomy. 42nd ed. Amsterdam, Holland: Elsevier.

[45] Taunton JE, Ryan MB, Clement DB, McKenzie DC, Lloyd-Smith DR and Zumbo BD (2002). A retrospective case-control analysis of 2002 running injuries. British Journal of Sports Medicine, 36(2), pp. 95-101.

[46] Wearing SC, Smeathers JE, Urry SR, Hennig EM and Hills AP (2006). The pathomechanics of plantar fasciitis. Sports Medicine (Auckland, N.Z.), 36(7), pp. 585-611.

[47] Welk AB, Haun DW, Clark TB and Kettner NW (2015). Use of high-resolution ultrasound to measure changes in plantar fascia thickness resulting from tissue creep in runners and walkers. Journal of Manipulative and Physiological Therapeutics, 38(1), pp. 81-85.

[48] Wilk BR, Fisher KL and Gutierrez W (2000). Defective running shoes as a contributing factor in plantar fasciitis in a triathlete. Journal of Orthopaedic \& Sports Physical Therapy, 30(1), pp. 21-31.

[49] Wong DW-C, Niu W, Wang Y and Zhang M (2016). Finite element analysis of foot and ankle impact injury: risk evaluation of calcaneus and talus fracture. PloS One, 11(4), p. e0154435.

[50] Wong DW-C, Wang Y, Leung AK-L, Yang M and Zhang M (2018). Finite element simulation on posterior tibial tendinopathy: Load transfer alteration and implications to the onset of pes planus. Clinical Biomechanics, 51(Supplement C), pp. 10-16.

[51] Zhang G M and Batra R C (2009). Symmetric smoothed particle hydrodynamics (SSPH) method and its application to elastic problems. Comput Mech, 43, pp. 321-340.

[52] Zhang M and Mak AF (1999). In vivo friction properties of human skin, Prosthetics and Orthotics International, 23(2), pp. 135-141. 